\title{
Gallium data variability and KamLAND
}

\author{
$\underline{\text { João Pulido, }}{ }^{\mathrm{a} *}$ Bhag Chauhan, ${ }^{\mathrm{a}}$ Marco Picariello ${ }^{\mathrm{b}}$ \\ ${ }^{a}$ Centro de Física Teórica das Partículas, Avenida Rovisco Pais P-1049-001, Lisboa, Portugal \\ ${ }^{\mathrm{b}}$ Dipartimento di Fisica, Università di Lecce, \\ Via Arnesano, ex Collegio Fiorini, I-73100 Lecce, Italia
}

We present an alternative fit to the conventional solar+KamLAND one which takes into account the possible time dependence of the Ga data and relies on the partial conversion of active $\rightarrow$ sterile neutrinos via the magnetic moment/solar field interaction. We evaluate the prediction for the solar neutrino rates obtaining a fit of similar quality as the LMA one. We also evaluate the KamLAND antineutrino survival probability as a function of reactor distance and find a better agreement with data as compared to LMA.

1. Time modulation of the solar neutrino flux is probably the most important issue after LMA has been asserted as the dominant solution to the solar neutrino problem. Many efforts have been undertaken recently by the experimental collaborations and theoretical groups 1233 to look into modulation. If this is confirmed it will probably imply the existence of a sizable neutrino magnetic moment $\mu_{\nu}$ and hence a wealth of new physics. The idea of neutrinos interacting with the solar magnetic field through their magnetic moment was first introduced in 1970 [4], revived in 1986 in connection to solar activity [5] and later viewed in terms of a resonant mechanism - the resonant spin flavour precession (RSFP) 677. In summary active $\nu_{e}^{\prime} s$ produced in the sun are assumed to be converted to sterile ones owing to the above interaction so that at times of intense solar activity a strong field leads to a large conversion with little or no conversion otherwise. Hence a neutrino flux anticorrelated to solar activity.

2. The Gallium solar neutrino data have been consistently decreasing in time, a striking fact that may be the signature of a long term periodicity. In fact, as can be seen from table 1, there is a $2.4 \sigma$ discrepancy in the combined results over the two periods which may be an indication of a possible anticorrelation of the Ga rate with the 11-year solar cycle. Ga are the only experiments

*email: pulido@cftp.ist.utl.pt with a significant contribution from $p p,{ }^{7} \mathrm{Be}$ neutrinos (together they account for $\simeq 80 \%$ of the event rate and more than $99 \%$ of the total solar flux). No other experiment shows such a variational effect, so the time dependence of the low energy fluxes becomes an open possibility. On the contrary, averaging Ga rates completely over time may erase important information already contained in the data. Hence we propose an alternative to the conventional solar+KamLAND fit attempting instead at two separate fits to the two Ga data sets consistent with all other solar and KamLAND data.

3. Our model 89] introduces light sterile neutrinos which only communicate to active ones through one magnetic moment interaction, so that in the vacuum

$$
\left(\begin{array}{l}
\nu_{s} \\
\nu_{e} \\
\nu_{x}
\end{array}\right)=\left(\begin{array}{ccc}
1 & 0 & 0 \\
0 & c_{\theta} & s_{\theta} \\
0 & -s_{\theta} & c_{\theta}
\end{array}\right)\left(\begin{array}{c}
\nu_{0} \\
\nu_{1} \\
\nu_{2}
\end{array}\right)
$$

and in matter

$\mathcal{H}_{\mathrm{M}}=\left(\begin{array}{ccc}\frac{-\Delta m_{10}^{2}}{2 E} & \mu_{\nu} B & 0 \\ \mu_{\nu} B & \frac{\Delta m_{21}^{2}}{2 E} s_{\theta}^{2}+V_{e} & \frac{\Delta m_{21}^{2}}{4 E} s_{2 \theta} \\ 0 & \frac{\Delta m_{21}^{2}}{4 E} s_{2 \theta} & \frac{\Delta m_{21}^{2}}{2 E} c_{\theta}^{2}+V_{x}\end{array}\right)$

where in (1) $\nu_{S}$ denotes the sterile neutrino and $V_{e}, V_{x}$ are the refraction indices. The parameter $\Delta m_{10}^{2}=m_{1}^{2}-m_{0}^{2}$ dictates the location of the active $\rightarrow$ sterile transition. It is plausible that the 
Table 1

Average rates for Ga experiments in SNU

\begin{tabular}{lll}
\hline Period & $1991-97$ & $1998-03$ \\
\hline SAGE+Ga/GNO & $77.8 \pm 5.0$ & $63.3 \pm 3.6$ \\
Ga/GNO only & $77.5 \pm 7.7$ & $62.9 \pm 6.0$ \\
SAGE only & $79.2 \pm 8.6$ & $63.9 \pm 5.0$ \\
av. no. of suspots & 52 & 100 \\
\hline
\end{tabular}

solar magnetic activity, as evidenced by sunspots, may extend down to the bottom of the convective zone at $x=0.71$ of the solar radius. Furthermore it is at the tachocline (the region extending from approximately $x=0.68$ to $x=0.72$ ) that the magnetic field is supposed to be peaked, this peak being closely connected with the local maximum of the angular velocity gradient. Therefore a 'plausible' magnetic field will be peaked around $x=0.71$ as shown in fig.1 of [9], and we assume the height of this peak to be closely connected to the solar activity. Since maximum conversion occurs near the resonance, low energy neutrinos are expected to resonate around this region and their flux may reflect the solar magnetic activity. Hence we need $\Delta m_{10}^{2}=O\left(10^{-8} \mathrm{eV}^{2}\right)$, thus excluding conversion to active neutrinos for which both possible values of the mass square difference are larger. The large order of magnitude discrepancy between $\Delta m_{10}^{2}$ and the other two implies the LMA and the spin flavour precession resonance to be located far apart, thus precluding their interference.

4. For typical parameter values

$B_{\text {peak }}=280 k G$

$\Delta m_{10}^{2}=-1.7 \times 10^{-8} \mathrm{eV}^{2}$

$\Delta m_{21}^{2}=8.2 \times 10^{-5} \mathrm{eV}^{2}$

$\tan ^{2} \theta=0.31$

we obtain the predictions shown in table 2 where the first two rows refer to the two consecutive periods in table 1 . We made use of the comparatively large uncertainty in the ${ }^{7} \mathrm{Be}$ flux from solar models [10] and assumed the normalizing factor $f_{B e}=1.1$. For comparison we recall the LMA best fit parameters for KamLAND only

$\Delta m_{21}^{2}=7.9 \pm_{0.5}^{0.6} \times 10^{-5} \mathrm{eV}^{2}, \tan ^{2} \theta=0.46 \pm_{0.25}^{4.5}(2 \sigma) .(7)$

5. Comparison of table 2 and eqs.(3)-(6) with fig.4 of ref.11] shows that the fit presented here lies well within the $95 \% \mathrm{CL}$ of the KamLAND best fit but just outside the $99.73 \% \mathrm{CL}$ of the solar fit. Such a result is to be expected since we neglected the conventional solar fit. We have for the global $\chi^{2}$ (excluding KamLAND):

$\chi^{2}=96.6 / 94$ d.o.f., $\chi_{L M A}^{2}=89.8 / 93$ d.o.f.

We have also evaluated the antineutrino survival probability for our model parameters $\Delta m_{21}^{2}$ and $\tan ^{2} \theta$ as given above [eqs.(4),(5)] as a function of effective reactor distance and compared it with the standard LMA one. This is given by the well known expression

$P_{o s c}\left(E_{\bar{\nu}}, L\right)=1-\sin ^{2} \theta \sin ^{2}\left(\frac{\Delta m_{21}^{2} L}{4 E_{\bar{\nu}}}\right)$

and the results are shown in fig.1. For the average reactor distance to which the data refer $(180$ $\mathrm{km}$ ) 11] we find, as seen from fig.1, $P=0.628$ and for the LMA case $P_{L M A}=0.578$ while the KamLAND collaboration quotes $P=0.658 \pm$ 0.044 (stat) \pm 0.047 (syst).

6. To conclude, we have investigated the consequences of a possible decrease of the Gallium event rate consistently observed by the two Gallium experiments as a function of time and related this decrease to solar activity. Our main motivation arises from the fact that no other experiment exhibits such a data variation and the Gallium event rate is the only one with a strong dependence on the low energy (LE) neutrino flux from the sun. Investigating the variability of the solar neutrino flux is the most important challenge facing us in solar neutrino physics, now that LMA is known to play a major role in the solution to the solar neutrino problem. A clear 
Table 2

The two sets of rates for Ga experiments in SNU. For comparison we list the LMA case.

\begin{tabular}{|c|c|c|c|c|c|c|c|c|c|}
\hline & $\mathrm{Ga}$ & $\mathrm{Cl}$ & $\mathrm{K}(\mathrm{SK})$ & $\mathrm{SNO}_{\mathrm{NC}}$ & $\mathrm{SNO}_{\mathrm{CC}}$ & $\mathrm{SNO}_{\mathrm{ES}}$ & $\chi_{\text {rates }}^{2}$ & $\chi_{S K_{s p}}^{2}$ & $\chi_{S N O_{g l}}^{2}$ \\
\hline$\overline{\text { Period 1991-97 }}$ & 73.8 & 2.72 & 2.29 & & & & 2.78 & & \\
\hline Period 1998-03 & 60.3 & & 2.28 & 5.65 & 1.59 & 2.25 & 0.54 & 47.5 & 48.5 \\
\hline LMA & 64.8 & 2.74 & 2.30 & 5.10 & 1.75 & 2.28 & 0.95 & 45.7 & 43.1 \\
\hline
\end{tabular}

distinction between our scenario and the LMA one will only be possible either with data improvement from KamLAND or average reactor distances below $110-120 \mathrm{~km}$ as can be seen from fig.1, or both. Interestingly enough our scenario based on LMA+RSFP gives an antineutrino survival probability at the average reactor distance of $180 \mathrm{~km}$ in better agreement with data than the LMA case. Important evolutions in KamLAND are expected: new reactors may come into operation while others cease and fluxes almost constantly change. This will provide us new opportunities by changing effective reactor distance thereby providing a distinction between the two scenarios investigated. Also the variability of the LE solar neutrino flux, possibly in connection to solar activity, will also be tested by the forthcoming LE experiments: KamLAND (solar mode), LENS, Borexino and SNO+.

\section{REFERENCES}

1. B. Aharmim et al. [SNO Collaboration], Phys. Rev. D 72, 052010 (2005) arXiv:hep-ex/0507079.

2. J. Yoo et al. [Super-Kamiokande Collaboration], Phys. Rev. D 68 (2003) 092002 arXiv:hep-ex/0307070.

3. P. A. Sturrock and D. O. Caldwell, Astrophys. J. 605, $568 \quad$ (2004) arXiv:hep-ph/0309239.

4. A. Cisneros, Astrophys.Space Sci.10:8792,1971.

5. L. B. Okun, M. B. Voloshin and M. I. Vysotsky, Sov. Phys. JETP 64 (1986) 446 [Zh. Eksp. Teor. Fiz. 91 (1986) 754].

6. C. S. Lim and W. J. Marciano, Phys. Rev. D 37, 1368 (1988).

7. E. K. Akhmedov, Phys. Lett. B 213, 64 (1988).

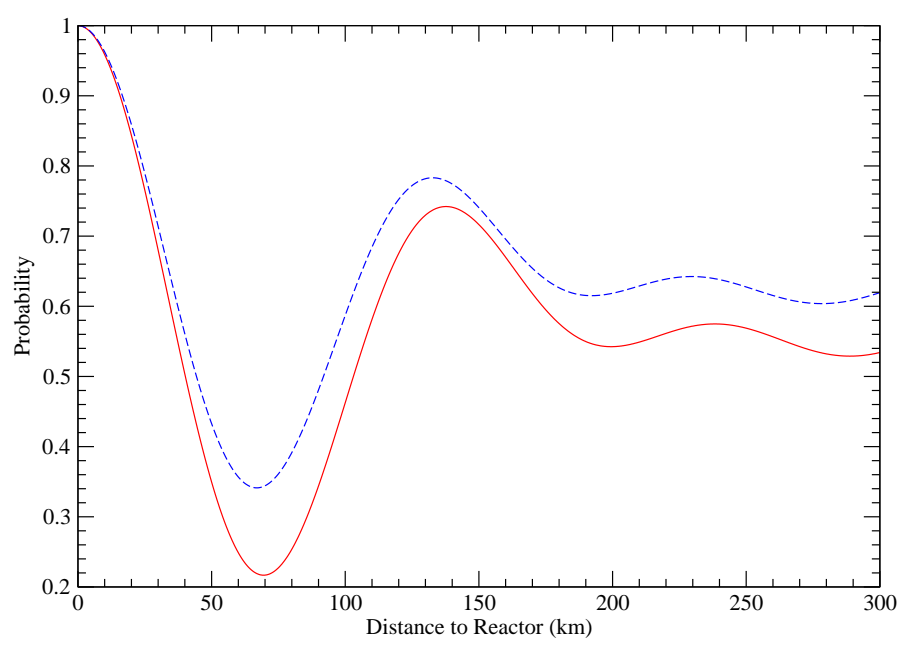

Figure 1. Survival probabilities for LMA (solid line) and our model parameters [eqs.(4),(5)] (dashed line).

8. B. C. Chauhan and J. Pulido, JHEP 0406, 008 (2004) arXiv:hep-ph/0402194.

9. B. C. Chauhan, J. Pulido and R. S. Raghavan, JHEP 0507 (2005) 051 arXiv:hep-ph/0504069.

10. A. Serenelli, these Proceedings.

11. T. Araki et al. [KamLAND Collaboration], Phys. Rev. Lett. 94 (2005) 081801 arXiv:hep-ex/0406035. US Patent No. 3904 422 (1975). 